\title{
Nuevos registros de bivalvos para el Mar Peruano
}

\author{
New records of bivalve from Peruvian Sea
}

Carlos Paredes* y Franz Cardoso

Presentado: $13 / 10 / 2003$

Aceptado: $\quad 07 / 11 / 2003$

\section{Resumen}

Seis especies de bivalvos: Thyasira flexuosa (Montagu, 1803); Diplodontina tumbesiana Stempell, 1899; Retrotapes exalbidus (Dillwyn, 1817); Pandora (Pandorella) cornuta C.B. Adams, 1852; Entodesma cuneatum (Gray, 1828); Periploma (Cricoploma) carpenteri Dall, 1896 se reportan por primera vez para el Mar Peruano. Se incluyen datos sobre la distribución, el hábitat y comentarios relevantes de cada una de las especies.

Palabras Claves: Bivalvos, Heterodonta, Anomalodesmata, nuevos registros, Perú.

\section{Abstract}

Six bivalve species: Thyasira flexuosa (Montagu, 1803); Diplodontina tumbesiana Stempell, 1899; Retrotapes exalbidus (Dillwyn, 1817); Pandora (Pandorella) cornuta C.B. Adams, 1852; Entodesma cuneatum (Gray, 1828); Periploma (Cricoploma) carpenteri Dall, 1896 are the first time reported for Peruvian sea. Distribution, habitat and relevant observations of each one species are included.

Key words: Bivalve, Heterodonta, Anomalodesmata, new records, Peru.

\section{Introducción}

Hace 30 años Marincovich (1973), comparando el número de especies de moluscos provistos de concha, conocidos entre Alaska y California, con el número de especies reportadas para la Provincia Peruana; y teniendo en cuenta que ambas regiones son similares en extensión y diversidad ambiental, postuló una mayor riqueza en la malacofauna de esta última. En consecuencia, hizo ver la necesidad de intensificar los inventarios en las costas del Perú y Chile. Sin duda la falta de especialistas y de muestreos continuos en las playas y orillas así como en el sublitoral, son las causas de que esta situación aún subsista.

Desde que Alamo y Valdivieso $(1987,1997)$ y Paredes et al. (1999) pusieron al día los registros de moluscos marinos del Perú, con un total de 1017 especies, incluyendo los que carecen de concha, los nuevos hallazgos son cada vez

\footnotetext{
* Laboratorio de Biología y Sistemática de Invertebrados Marinos, Facultad de Ciencias Biológicas, UNMSM, Apdo. 05D-51, Lima 1, Perú, y Departamento de Malacología, Museo de Historia Natural, UNMSM, Apdo. 14-0434, Lima 14, Perú.

E-mail Carlos Paredes: cparedesq@unmsm.edu.pe
}

más frecuentes (Mogollón et al., 1999, 2000; Paredes y Cardoso, 1999, 2001a, b; 2003). En esta ocasión se informa por primera vez de la presencia de seis especies de moluscos bivalvos para el mar peruano, una fue hallada en la zona de transición de la Provincia Panameña y cinco fueron colectadas en aguas de la Provincia Peruana.

\section{Material y métodos}

La colecta del material se realizó a mano en la zona intermareal y mediante dragado o buceo autónomo en el infralitoral, durante los trabajos de campo programados en nuestras actividades de investigación sobre la diversidad de la malacofauna peruana. Periploma carpenteri fue colectada durante el Crucero BIC Humboltd 9607-08. El material fue fijado con formol al $7 \%$ neutralizado con bórax y conservado en alcohol etílico al 70\%. Para la determinación taxonómica se utilizó la bibliografía especializada y se contó con el apoyo del doctor Eugene Coan del Museo de Historia Natural de Santa Barbara (SBMNH). Para el ordenamiento sistemático se siguió a Coan 


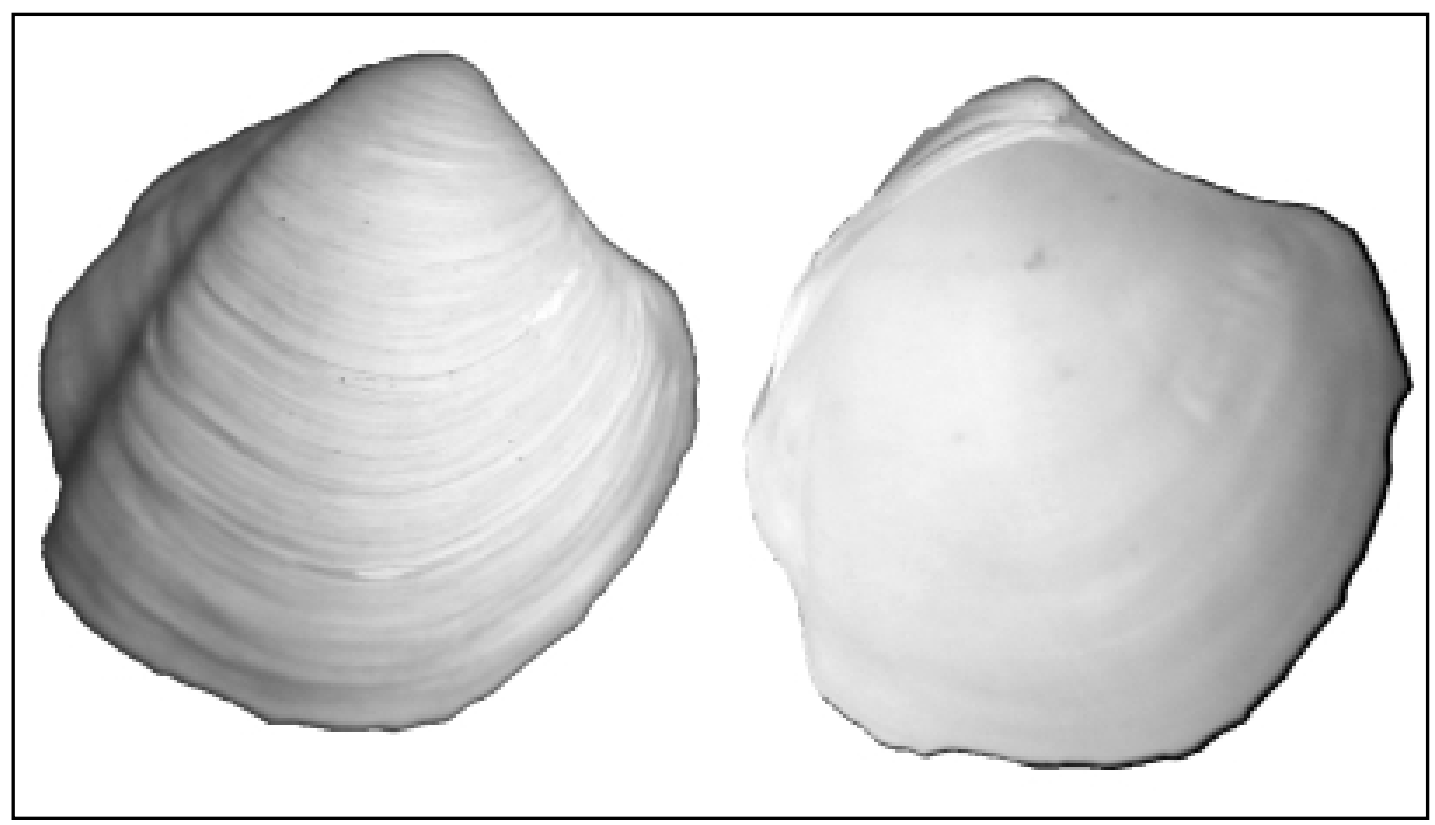

Figura 1. Thyasira flexuosa, longitud $13,0 \mathrm{~mm}$.

et al (2000). El material está depositado en las colecciones del Museo de Historia Natural de la Universidad Nacional Mayor de San Marcos (MUSM) y el Laboratorio de Biología y Sistemática de Invertebrados Marinos de la Facultad de Ciencias Biológicas de la Universidad Nacional Mayor de San Marcos (LaBSIM).

\section{Resultados}

$\begin{array}{lc}\text { SubClase } & \text { HetERODONTA } \\ \text { ORDEN } & \text { VENEROIDA } \\ \text { SuPERFAMILIA } & \text { LuCINOIDEA FlEMING, 1828 } \\ \text { FAMILIA } & \text { ThyASIRIDAE DALL, 1900 } \\ \text { SubFAMILIA } & \text { ThyASIRINAE DALL, 1900 }\end{array}$

\section{Thyasira flexuosa (Montagu, 1803)}

(Figura 1)

Tellina flexuosa Montagu, 1803: 72.

Thyasira barbarensis, Keen 1971: 131, fig. 301.

Thyasira (Thyasira) barbarensis, Bernard 1983: 29.

Thyasira flexuosa, Coan et al., 2000: 275-276, pl. 53.

Material examinado: 1 lote, 2 ejemplares, 1 valva; 18-08-1986; LaBSIM.

Descripción: Concha delgada y frágil más alta que larga, subcuadrangular inflada, con umbos prominentes prosogirados; lúnula romboidal sin contorno definido; escudo alargado y estrecho; extremo posterior con un surco radial que se inicia angosto cerca del pico y se expande hasta el borde de la concha; lámina articular estrecha y sin dientes; escultura concéntrica presenta finas estrías y costillas espaciadas poco desarrolladas. Coloración blanca con el interior aporcelanado.

Distribución: Circumboreal y Panarctica; Mar Beaufort, Alaska, a Punta Loma, California, y al Golfo de California; al sur hasta el Norte de África en el Atlántico este, a Florida en el Atlántico oeste, y a Hokkaido, Japón, en el Pacifico Oeste (Coan et al., 2000).

Nueva localidad: Ica (Bahía Independencia).

Hábitat: Fango fino, $32 \mathrm{~m}$.

Comentarios: Según Coan et al. (2000), la forma de esta especie es muy variable, lo cual dificulta la diagnosis. Los ejemplares fueron determinados por Paul Scott (SBMNH).

$\begin{array}{lc}\text { Subclase } & \text { Heterodonta } \\ \text { Orden } & \text { VenEROIDA } \\ \text { SuPERFAMILIA } & \text { GaLeOMMATOIDEA Gray, 1840 } \\ \text { FAMILIA } & \text { LASAEIDAE GRAY, } 1842\end{array}$

Diplodontina tumbesiana Stempell, 1899

(Figura 2) 

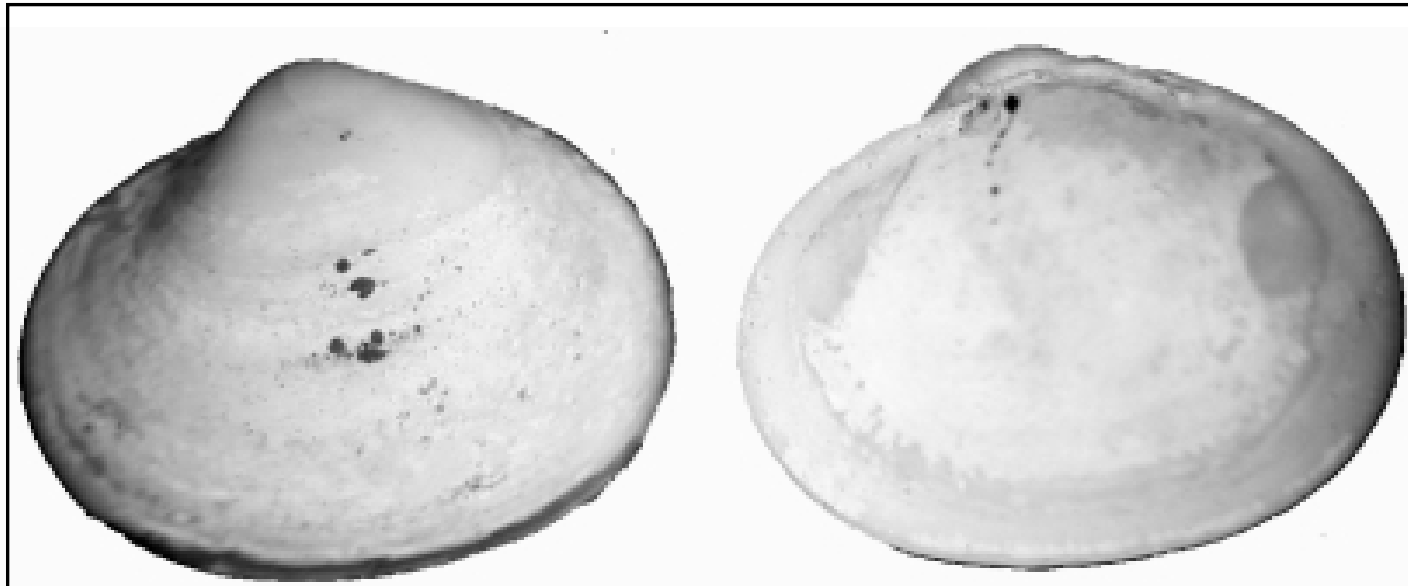

Figura 2. Diplodontina tumbesiana, longitud 9,6 mm.

Diplodontina tumbesiana Stempell, 1899:232, pl. 12, figs. 18, 19a; Bernard, 1983:31.

Kellia tumbesiana, Dall, 1909:264; Soot-Ryen, 1959:50, fig. 4a; Marincovich, 1973:12, figs. 1214; Guzmán et al. 1998:67-68, fig.8.

Material examinado: 1 lote, 1 ejemplar (solo valvas); 11-09-2003; LaBSIM.

Descripción: Concha ovalada con umbo prominente subcentral y prosogirado; dientes cardinales bien desarrollados en ambas valvas, el lateral posterior de la valva derecha es más desarrollado que el de la valva izquierda; las líneas de crecimiento casi no se aprecian por el deterioro de la conchilla; la línea paleal entera y las huellas de los músculos aductores son nítidos. Coloración general blanquecina.

Distribución: Iquique a Cobija, Chile (Marincovich, 1973).

Nueva localidad: Ica (San Juan de Marcona, Playa Guaneras).

Hábitat: Varado en la playa pedregosa.

Comentarios: Guzmán et al. (1998) confunden la distribución de esta especie, puesto

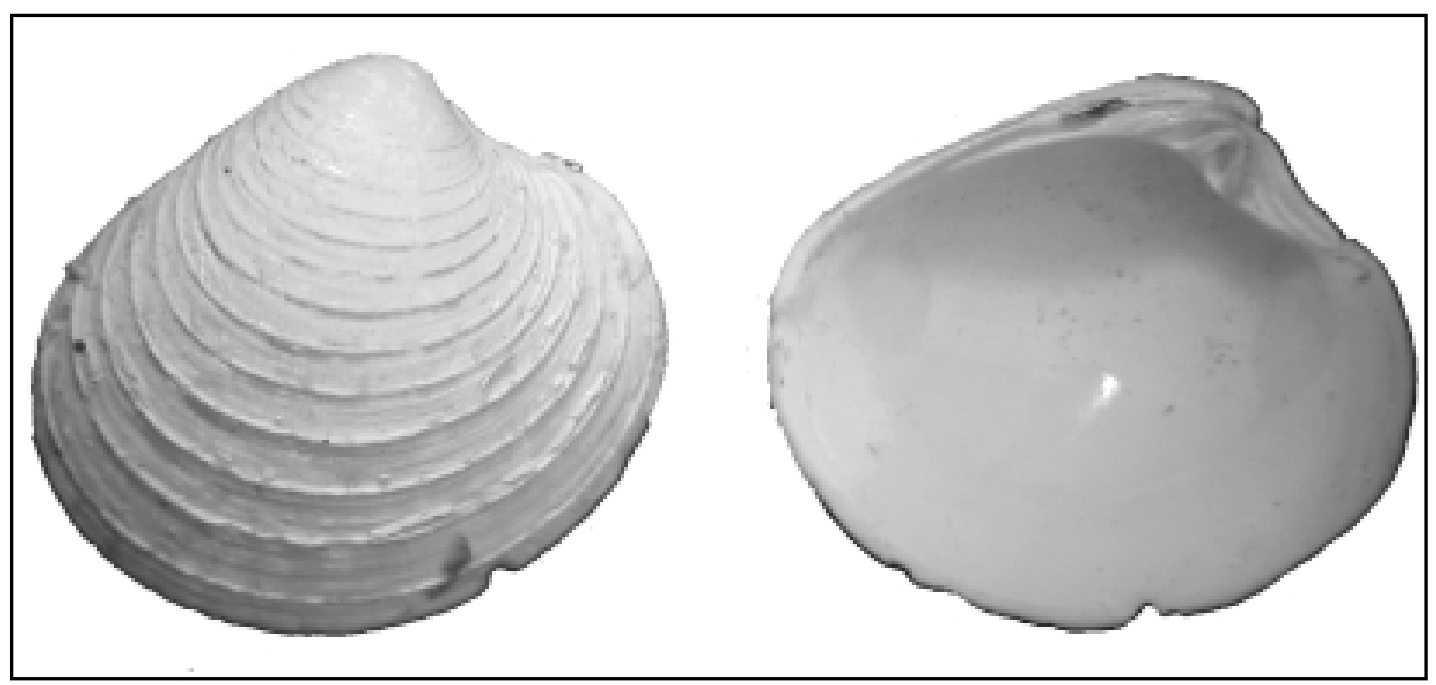

Figura 3. Retrotapes exalbidus, longitud 7,4 mm. 
que Dall (1909) no la reportó para Perú, sino para «Tumbes Peninsula near Talcahuano, Chile».

\begin{tabular}{|c|c|}
\hline Subclase & HETERODONTA \\
\hline ORDEN & VENEROIDA \\
\hline SUPERFAMILIA & VENEROIDEA RAFINESQUE, 1815 \\
\hline FAMILIA & VENERIDAE RAFINESQUE, 1815 \\
\hline SUBFAMILIA & TAPETINAE GRAY, 1853 \\
\hline
\end{tabular}

\section{Retrotapes exalbidus (Dillwyn, 1817)}

(Figura 3)

Venus exalbida Dillwyn, 1817: 170.

Katelsia (Samarangia) exalbida, Riveros-Zuñiga y González 1950: 139-141, fig. 31.

Samarangia exalbida, Carcelles y Williamson 1951: 342.

Eurhomalea exalbida, Soot-Ryen 1959: 59; Castellanos, 1967: 250-251,pl. 22, figs. 4-5; Osorio y

Bahamonde 1970: 203; Bernard, 1983: 55.

Retrotapes exalbida, del Rio 1997: 80, 82, figs. 22$24,41$.

Retrotapes exalbidus, Reid y Osorio 2000: 139, fig. 5J.

Material examinado: 1 lote, 2 ejemplares juveniles; 29-08-1986; LaBSIM.

Descripción: Concha subquadrada con umbo cerca del extremo anterior redondeado; extremo posterior subtruncado; lúnula definida frente a los picos; escultura con lamelas concéntricas bien separadas; charnela con 3 dientes cardinales fuertes en cada valva; margen interno liso. Coloración externa cremosa, interior blanco.

Distribución: Isla Chilóe hasta Cabo de Hornos, Chile (Reid y Osorio, 2000), y en el Atlántico hasta el Mar del Plata, Argentina (Carcelles y Willianson, 1951).

Nueva localidad: Ica (Bahía Independencia).

Habitat: Fondo blando, 14 m.

Comentarios: Esta especie ha sido asignada a un nuevo género Retrotapes por del Rio (1997).

\begin{tabular}{|c|c|}
\hline SubClase & ANOMALODESMATA \\
\hline ORDEN & PHOLADOMYOIDA \\
\hline ERFAMILIA & ROIDEA RAFINESQUE, \\
\hline FAMILIA & PANDORIDAE RAFINESQUE, 1815 \\
\hline
\end{tabular}

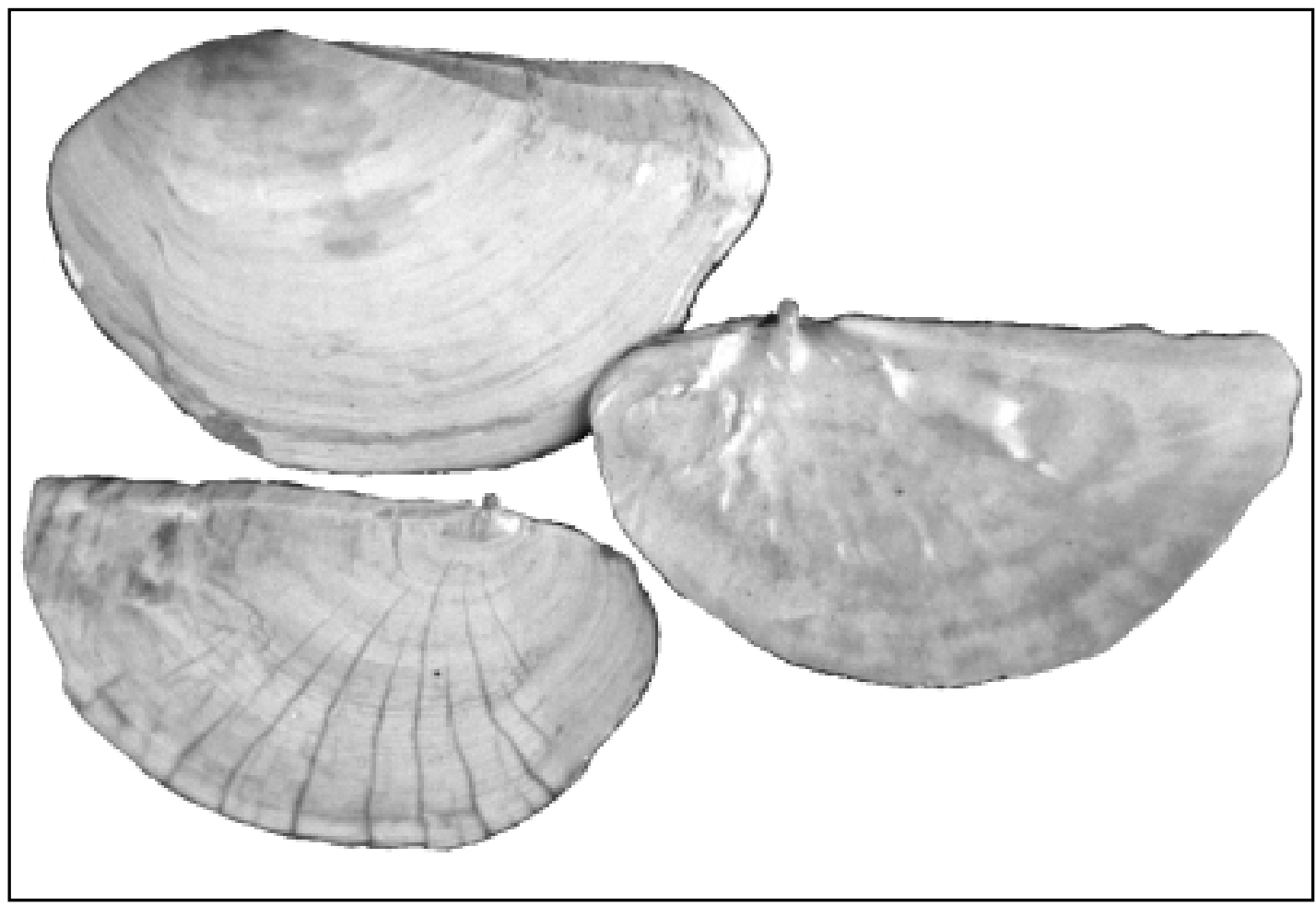

Figura 4. Pandora (Pandorella)cornuta, longitud 22,2 mm 


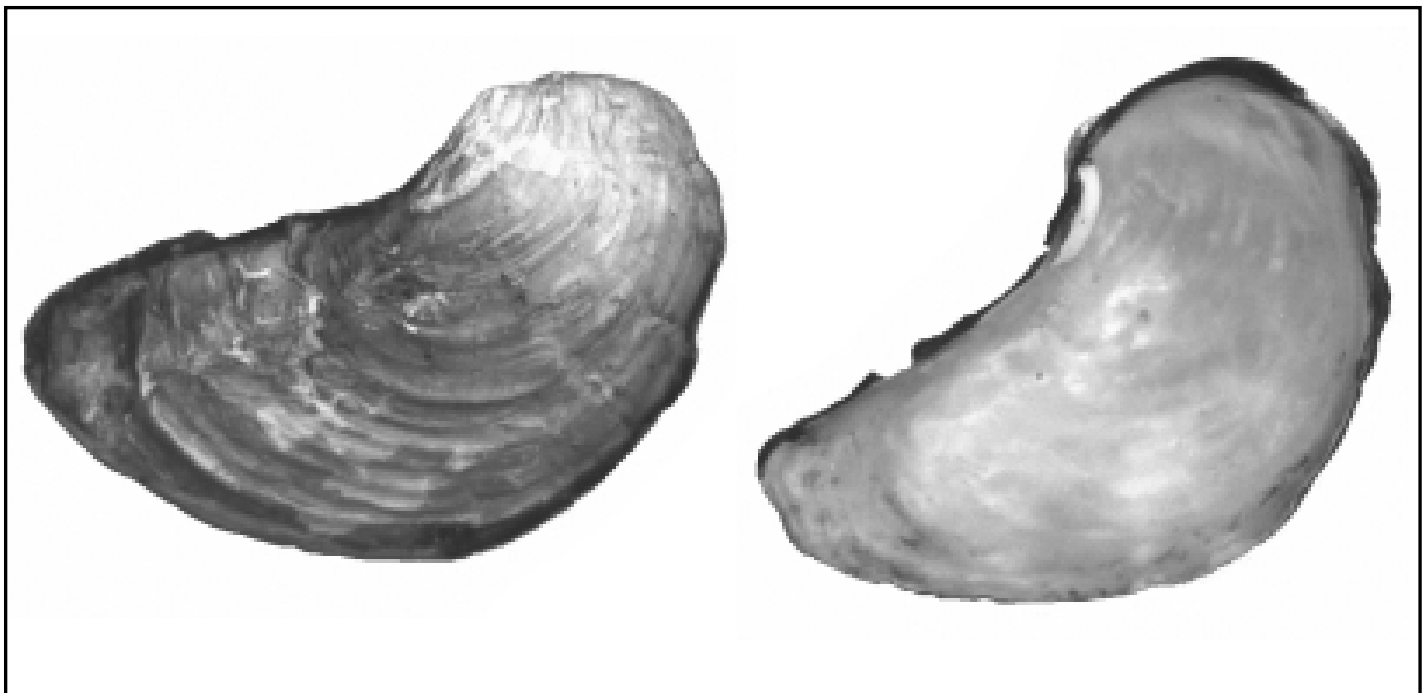

Figura 5. Entodesma cuneatum, longitud $36,8 \mathrm{~mm}$

Pandora (Pandorella) cornuta C.B. Adams, 1852

(Figura 4)

Pandora cornuta C.B. Adams, 1852: 519, 547.

Pandora (Pandorella) cornuta, Olsson 1961: 455, pl.81, figs. 7, 7a; Keen 1971: 289, fig. 738; Bernard, 1983: 63; Skoglund, 1991: 52.

Material examinado: 1 lote, 4 ejemplares, 2 valvas; 08-08-1986; MUSM.-1 lote, 4 ejemplares, 1 valva; 18-08-1986; MUSM.-1 lote, 1 ejemplar; 06-07-1987; LaBSIM.

Descripción: Concha semicircular con el umbo cerca del extremo anterior redondeado; extremo posterior alargado con el margen dorsal casi recto; valvas desiguales, la izquierda convexa y la derecha aplanada. En la valva izquierda hay un dentículo debajo del pico; una ligera depresión radial separa el disco de la vertiente anterior; la escultura consiste en costillas radiales casi obsoletas en el disco y tres costillas radiales notorias cerca del borde dorsal posterior ligeramente arqueado. Valva derecha con hendiduras radiales finas y una costilla cerca del margen dorsal posterior recto. Coloración blanquecina con interior subnacarado.

Distribución: Panamá (Bernard, 1983).

Nueva localidad: Ica (Bahía Independencia).
Hábitat: Sublitoral en fondo blando, $31 \mathrm{~m}$.

Comentarios: Las ilustraciones de Olsson (1961), reproducidas por Keen (1971)y las descripciones son incompletas, lo cual permite algunas dudas; sin embargo, el Dr. Eugene Coan (com. pers. 2003) considera que se trata de $P$. cornuta. Creemos que la especie se podría confundir con $P$. (Foveadens) panamensis Dall, 1915 , pero esta última presenta la depresión radial en el disco y el borde posterior interno de la valva izquierda no tiene las características que se ven en la figura de Olsson (1961: pl. 81, fig. 2). Con este registro, son dos las especies de Pandora halladas en el Perú.

$\begin{array}{lc}\text { SubClase } & \text { ANOMALODESMATA } \\ \text { ORdEN } & \text { PhOLADOMYOIDA } \\ \text { SuPERFAMILIA } & \text { PANDOROIDEA RAFINESQUE, 1815 } \\ \text { FAMILIA } & \text { LyONSIIDAE FISCHER, 1887 }\end{array}$

\section{Entodesma cuneatum (Gray, 1828)}

(Figura 5)

Anatina cuneata Gray, 1828: 6, pl.3, fig. 14.

Entodesma cuneata, Dall 1909: 259; Carcelles y Williamson, 1951: 348; Soot-Ryen, 1959: 35; Osorio y Bahamonde 1970: 195; Dell, 1972: 29-30, fig. 23; Marincovich, 1973: 15, fig. 25.

Entodesma (Entodesma) cuneatum, Bernard 1983: 63. 


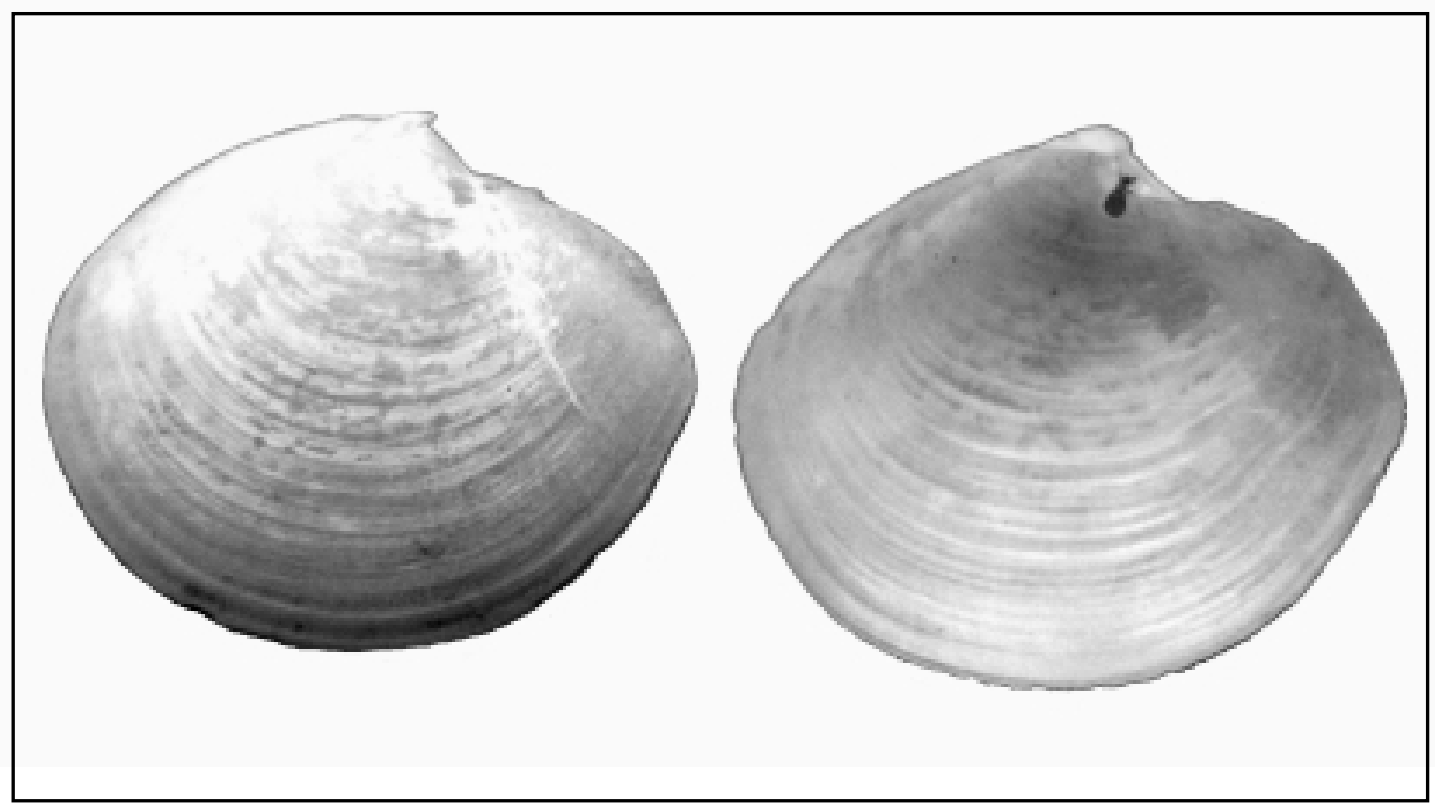

Figura 6. Periploma (Cricoploma)carpenteri, longitud $25,7 \mathrm{~mm}$

Material examinado: 1 lote, 1 ejemplar; 12-10-1992; MUSM.-1 lote, 2 ejemplares; 2605-2002; LaBSIM.

Descripción: Concha de forma irregular con umbo cerca del extremo anterior, inflada en la región del disco y con el borde anterior redondeado; extremo posterior alargado con las valvas entreabiertas; charnela sin dientes, condróforo pequeño y litodesma grande; escultura con ondulaciones concéntricas que se reflejan internamente. Periostraco de color pardo y color de la concha blanquecino con el interior subnacarado.

Distribución: Pisagua hasta Puerto Montt, Chile (Bernard, 1983).

Nuevas localidades: Ica (Bahia Independencia), Moquegua (Playa tres Hermanas).

Hábitat: En rizoides del alga Lessonia sp.

Comentarios: Nuestro ejemplar presenta el extremo posterior más alargado que el ilustrado por Marincovich (1973).El límite norte dado por Dall (1909) ha sido cuestionado.

\begin{tabular}{|c|c|}
\hline SUBCLASE & ANOMALODESMATA \\
\hline ORDEN & PhOLADOMYOIDA \\
\hline SUPERFAMILIA & Thracioidea Stoliczka, 1870 \\
\hline FAMILIA & PERIPLOMATIDAE DALL, 1895 \\
\hline
\end{tabular}

\section{Periploma (Cricoploma) carpenteri Dall, 1896}

(Figura 6)

Periploma carpenteri Dall, 1896: 20

Periploma (Periploma) carpenteri, Olsson 1961: 462, pl.82, fig. 7, 7a; Keen, 1971: 292, fig. 749; Bernard, 1983: 64.

Material examinado: 1 lote, 1 ejemplar, 28-07-1996; MUSM.- 1 lote, 1 ejemplar, 0208-1996; LaBSIM.

Descripción: Concha orbicular, frágil, con umbo subcentral opistogirado y con fisura radial; extremo anterior redondeado y posterior ligeramente anguloso con brecha; lámina articular delgada, condróforo sostenido por una costilla, litodesma presente; superficie de la concha con finas costillas concéntricas y pequeños gránulos que son más conspicuos en la mitad inferior. Coloración blanca con interior ligeramente nacarado. 
Distribución: El Salvador hasta Bahía de Panamá (Olsson, 1961).

Nuevas localidades: Tumbes $\left(03^{\circ} 45.4^{\prime} \mathrm{S}\right.$, $81^{\circ} 09.1^{\prime} \mathrm{W}$; $\left.03^{\circ} 48.4^{\prime} \mathrm{S}, 81^{\circ} 10.8^{\prime} \mathrm{W}\right)$.

Hábitat: Fondo blando, 389-412 m.

Comentarios: El ejemplar del 02-08-96 presenta la valva izquierda rota y mide 42,0 $\mathrm{mm}$ de longitud. Otra especie citada para el Perú es $P$. planiusculum Sowerby I, 1834, en aguas someras (Bernard, 1983).

\section{Literatura citada}

Adams, C.B. 1852. Catalogue of shells collected at Panama, with notes on synonymy, station, and habitat. Anns. Lyceum Nat. Hist. New York. 5: 229-566.

Alamo, V. y V. Valdivieso. 1987. Lista sistemática de moluscos marinos del Perú. Bol. Inst. Mar. Perú- Callao. Volumen Extraordinario: 205 pp. 1997. Lista sistemática de moluscos marinos del Perú. Segunda edición, revisada y actualizada. Publicación Especial. Bol. Inst. Mar. Perú- Callao. 1833 pp.

Bernard, F.R. 1976. Living Chamidae of the Eastern Pacific (Bivalvia: Heterodonta). Los Angeles Co. Mus. Nat. Hist. Contributions in Science 278: 1-43.

1983. Catalogue of the living bivalvia of the Eastern Pacific Ocean: Bering Strait to Cape Horn. Canadian Special Publication of Fisheries and Aquatic Sciences 61: 102 p.

Carcelles, A.R. y S.I. WILLIAMSON. 1951. Catálogo de los Moluscos Marinos de la Provincia Magallánica. Rev. Inst. Nac. de Inves. Cient. Nat Argentina). Cien. Zool. 2(5): 225-383.

Castellanos, Z. A. de. 1967. Catálogo de los Moluscos Marinos Bonaerenses. Anales de la Comisión Científica. Provincia de Buenos Aires, La Plata. $390 \mathrm{pp}$.

Coan, E.V., P. Scott y F. R. Bernard. 2000. Bivalve Seashells of Westewrn North America: Marine Bivalve Mollusks from Artic Alaska to Baja California. Santa Barbara Museum of Natural History Monographs Number 2: 764 p.

Dall, W.H. 1896. Diagnoses of new species of mollusks from the west coast of America. Proc. U.S. Natl. Mus. 18: 7-20.

1909. Report on a collection of shells from Perú, whith a summary of the littoral marine Mollusca of the Peruvian Zoological Province. Proc. U.S. Natl. Mus. 37(1704): 147-294.

Dell, R.K. 1972. Notes on nomenclatura of some Mollusca from Antarctica and southern South
America. Rec. Dominion Mus. (Wellington) 8(3): 21-42.

Dillwyn, L.W. 1817. A descriptive catalogue of Recent shells, arranged according to the Linnaean method: with particular attention to the synonymy. London 1: $580 \mathrm{pp}$.

Gray, J.E. 1828. Spicilegia zoológica; or, original figures and short systematic descriptions of new and unfigured animals. Part 1. London. 8 pp.

Guzmán, N., S. Saa y L. Ortlieb. 1998. Catálogo descriptivo de los moluscos litorales (Gastropoda y Pelecypoda) de la zona en Antofagasta, $23^{\circ} \mathrm{S}$ (Chile). Estud. Oceanol. 17: 17-86.

Keen, A.M. 1971. Sea shells of tropical West America. 2a. ed. Stanford Univ. Press, California, 1064 pp., 3325 figs. 22 pls.

Marincovich, L. 1973. Intertidal Mollusks of Iquique, Chile. Los Angeles Co. Mus. Nat. Hist., Sc. Bull. 16:1-49, 102 figs.

Mogollon, V., N. Chirichigno, L. Clemente y M. Peña. 1999. Gasterópodos (Mollusca) de Isla Los Chimus y alrededores (Santa, Ancash, Perú). Wiñay Yachay 3(2): 35-75.

Mogollon, V., M. Peña y P. Bearez. 2000. Nuevos registros de bivalves (Mollusca) en el mar del Perú. Wiñay Yachay 4(1): 77-95.

Montagu, G. 1803. Testacea Británica or Natural History of British Shells, marine, land, and fresh-water, including the most minute: systematically arranged and embellished with figures. London. $610 \mathrm{pp}$.

Olsson, A.A. 1961. Mollusks of the tropical eastern Pacific. Paleonto. Res. Ins. Ithaca, New york. 574 pp., pls 1-86.

Osorio, C. y N. Bahamonde. 1970. Lista preliminar de Lamelibranquios de Chile. Boletin del Museo Nacional de Historia Natural, Chile 31: 185-256.

Paredes, C y F. Cardoso. 1999. Nuevas adiciones de bivalvos a la malacofauna marina Peruana. Rev. peru. biol. 6(1): 48-53.

2001a. Nuevos registros de Protobranchia (Mollusca: Bivalvia) para el mar peruano. Rev. peru. biol. 8(1): 5-10.

. 2001b. El género Donax en la costa Peruana (Bivalvia: Tellinoidea). Rev. peru. biol. 8(2): 83-93.

2003. Adiciones a los Moluscos bivalvos marinos del Perú. Rev. peru. biol. 10(1): 53-58.

Paredes, C., P. Huaman, F. Cardoso, R. Vivar y V. Vera. 1999. Estado actual del conocimiento de los moluscos acuáticos en el Perú. Rev. Perú. biol. 6(1): 5-47.

Reid, D. y C. Osorio. 2000. The shallow-water marine Molusca of the Estero Elefantes and Laguna San Rafael, Southern Chile. Bull. Nat. Hist. Mus. Lond. (Zool.) 66(2): 109-146. 
Rio, C.J. del. 1997. Cenozoic biogeographic history of the eurythermal Genus Retrotapes, new genus (Subfamily Tapetinae) from southern South America and Antarctica. Nautilus 110: 77-93.

Riveros-Zuñiga, F. y J. Gonzalez. 1950. Catálogo descriptivo de venéridos Chilenos. Rev Biol. Mar. (Univ. Chile) 2(2/3):117-160, figs. text 22-44.
Skoglund, C. 1991. Additions to the Panamic Province Bivalve (Mollusca) literature 1971 to 1990. The Festivus 22(Suppl. 2 to 1990): 1-74.

Soot-Ryen, T. 1959. Pelecypoda. Repts. Lund Univ. Chile Expd. (1948-1949) 35: 1-86.

Stempell, W. 1899. Die Muscheln der Sammlung Plate. Zool. Jahrb. (Suppl. 4)2: 217-250. 